\title{
Asymptotic modelling of weakly twisted electrostatic problems
}

\author{
André Nicolet ${ }^{\mathrm{a}}$, Alexander B. Movchan ${ }^{\mathrm{b}, *}$, Sébastien Guenneau ${ }^{\mathrm{b}}$, Frédéric Zolla ${ }^{\mathrm{c}}$ \\ ${ }^{a}$ Institut Fresnel, UMR 6133, université Aix-Marseille III, case 162, 13397 Marseille cedex 20, France \\ ${ }^{\mathrm{b}}$ Department of Mathematical Sciences, Liverpool University, Liverpool L69 3BX, UK \\ ${ }^{\mathrm{c}}$ Institut Fresnel, UMR 6133, université Aix-Marseille I, case 162, 13397 Marseille cedex 20, France
}

Received 23 September 2005; accepted after revision 29 November 2005

Available online 9 February 2006

Presented by Pierre Suquet

\begin{abstract}
We present an analysis of an electrostatic field within a helicoidal structure with a twist, which is small compared to the characteristic size of the cross-section. The asymptotic results are checked against exact computations thanks to helicoidal coordinates, which preserve the intrinsically two-dimensional nature of the problem. The numerical studies are performed using the finite elements. To cite this article: A. Nicolet et al., C. R. Mecanique 334 (2006).
\end{abstract}

๑ 2006 Académie des sciences. Published by Elsevier SAS. All rights reserved.

\section{Résumé}

Modélisation asymptotique de problèmes électrostatiques faiblement torsadés. Nous proposons une analyse du champ électrostatique dans une structure torsadée par une approche de type perturbative, à la limite des faibles torsions. La méthode asymptotique décrite est validée à l'aide d'une modélisation exacte de la torsion qui préserve le caractère essentiellement bidimensionnel du problème (coordonnées hélicoïdales). Les études numériques sont menées en utilisant les éléments finis. Pour citer cet article $:$ A. Nicolet et al., C. R. Mecanique 334 (2006).

(C) 2006 Académie des sciences. Published by Elsevier SAS. All rights reserved.

Keywords: Acoustics; Waves; Vibrations; Helicoidal coordinates; Finite elements; Asymptotic methods; Twisted fibres; Accuracy

Mots-clés : Acoustique; Ondes; Vibrations ; Coordonnées hélicoïdales ; Éléments finis ; Méthodes asymptotiques ; Fibres torsadées ; Précision

\section{Version française abrégée}

Cette Note présente un modèle asymptotique et numérique d'un problème de type Dirichlet dans un domaine cylindrique contenant des canaux hélicoïdaux. On entend par «canal hélicoïdal» et plus généralement «structure torsadée», une structure d'extension infinie dans une direction et qui est invariante selon cette direction suivant les coordonnées locales données en (2). Dans toute sa généralité ce problème tridimensionnel est assez délicat et se traite numériquement par une méthode de type éléments finis. On utilise, ici, un algorithme qui permet de réduire le

\footnotetext{
* Corresponding author.

E-mail addresses: andre.nicolet@fresnel.fr (A. Nicolet), abm@liv.ac.uk (A.B. Movchan), guenneau@liverpool.ac.uk (S. Guenneau), frederic.zolla@fresnel.fr (F. Zolla).
} 
problème à un problème bidimensionnel par le truchement des coordonnées locales, non-orthogonales (2), puis dans un deuxième temps, on propose un algorithme asymptotique simple à mettre en œuvre qui met à profit le caractère faiblement torsadé des canaux hélicoïdaux. Voici comment on procède.

Pour obtenir la formulation asymptotique, on utilise l'Ansatz suivant

$$
u_{\eta}=\sum_{k=0}^{\infty} \eta^{2 k} u_{k}\left(\frac{x_{1}}{\eta}, \frac{x_{2}}{\eta}, x_{3}\right) \sim u_{0}\left(\frac{x_{1}}{\eta}, \frac{x_{2}}{\eta}, x_{3}\right)+\eta^{2} u_{1}\left(\frac{x_{1}}{\eta}, \frac{x_{2}}{\eta}, x_{3}\right)
$$

où $\eta$ est un petit paramètre adimensionné qui est lié au rapport du diamètre de la section droite sur la période de la torsade. Les fonctions $u_{0}$ et $u_{1}$ dépendant des variables transverses rapides $\left(y_{1}=\frac{x_{1}}{\eta}, y_{2}=\frac{x_{2}}{\eta}\right)$ sont obtenues comme solutions de problèmes bidimensionnels dans la section droite de la structure en hélice. Cette solution asymptotique a été éprouvée numériquement en la comparant avec l'approche générale « exacte » fondée sur les coordonnées hélicoïdales. Les résultats se sont avérés robustes et très précis y compris dans le cas de torsades relativement importantes.

La structure de l'article est la suivante : dans la première section nous présentons les aspects géométriques et définissons les coordonnées hélicoïdales et leurs propriétés. Dans la deuxième section, nous définissons le problème asymptotique pour une faible torsade et nous obtenons la solution approchée. Le schéma numérique prenant en compte la correction asymptotique est détaillé dans la troisième section. On se ramène à deux problèmes bidimensionnels : le problème (13) de type Laplace et le problème (14) de type Poisson où les sources dépendent de la solution du problème (13). On obtient ainsi un développement asymptotique de la solution qui dépend du paramètre $\alpha$ (qui décrit la torsion) de manière explicite. De façon assez remarquable la solution du problème pour $\alpha=1$ fournit aussi les solutions pour toute valeur acceptable de $\alpha$. Par la suite, nous donnons la forme faible du problème (14) qui permet sa résolution par une méthode de type éléments finis. Finalement, la quatrième section présente un exemple numérique détaillé et une discussion de la précision numérique de la méthode proposée.

\section{Geometric setting for twisted structures}

We consider an electrostatic problem in a twisted structure made of conducting and dielectric materials that can be described parametrically along a given axis in such a way that various cross-sections along this axis are just rotations of each others through an angle proportional to the distance between the sections. A typical example of a twisted structure is shown in Fig. 1.

For the sake of clarity of the presentation, we consider the case when the structure in question includes a single twisted fibre.

Let us introduce a co-ordinate system $\left(\xi_{1}, \xi_{2}, \xi_{3}\right)$ deduced from rectangular Cartesian co-ordinates $\left(x_{1}, x_{2}, x_{3}\right)$ in the following way

$$
\left\{\begin{array}{l}
x_{1}=\xi_{1} \cos \left(\alpha \xi_{3}\right)+\xi_{2} \sin \left(\alpha \xi_{3}\right) \\
x_{2}=-\xi_{1} \sin \left(\alpha \xi_{3}\right)+\xi_{2} \cos \left(\alpha \xi_{3}\right) \\
x_{3}=\xi_{3}
\end{array}\right.
$$

where $\alpha$ is a parameter which characterises the torsion of the structure. Conversely,

$$
\left\{\begin{array}{l}
\xi_{1}=x_{1} \cos \left(\alpha x_{3}\right)-x_{2} \sin \left(\alpha x_{3}\right) \\
\xi_{2}=x_{1} \sin \left(\alpha x_{3}\right)+x_{2} \cos \left(\alpha x_{3}\right) \\
\xi_{3}=x_{3}
\end{array}\right.
$$

A twisted structure is a structure for which both geometrical and physical characteristics (here $\varepsilon$ and the geometry of the conductors) only depend on $\xi_{1}$ and $\xi_{2}$.

This general co-ordinate system is characterised by the Jacobian of the transformation (1):

$$
\mathbf{J}=\frac{\partial\left(x_{1}, x_{2}, x_{3}\right)}{\partial\left(\xi_{1}, \xi_{2}, \xi_{3}\right)}=\left(\begin{array}{ccc}
\cos \left(\alpha \xi_{3}\right) & \sin \left(\alpha \xi_{3}\right) & \alpha \xi_{2} \cos \left(\alpha \xi_{3}\right)-\alpha \xi_{1} \sin \left(\alpha \xi_{3}\right) \\
-\sin \left(\alpha \xi_{3}\right) & \cos \left(\alpha \xi_{3}\right) & -\alpha \xi_{1} \cos \left(\alpha \xi_{3}\right)-\alpha \xi_{2} \sin \left(\alpha \xi_{3}\right) \\
0 & 0 & 1
\end{array}\right)
$$

which does depend on the three variables $\xi_{1}, \xi_{2}$ and $\xi_{3}$. On the contrary, the transformation matrix $\mathbf{T}[1,2]$

$$
\mathbf{T}\left(\xi_{1}, \xi_{2}\right)=\frac{\mathbf{J}^{T} \mathbf{J}}{\operatorname{det}(\mathbf{J})}=\left(\begin{array}{ccc}
1 & 0 & \alpha \xi_{2} \\
0 & 1 & -\alpha \xi_{1} \\
\alpha \xi_{2} & -\alpha \xi_{1} & 1+\alpha^{2}\left(\xi_{1}^{2}+\xi_{2}^{2}\right)
\end{array}\right)
$$




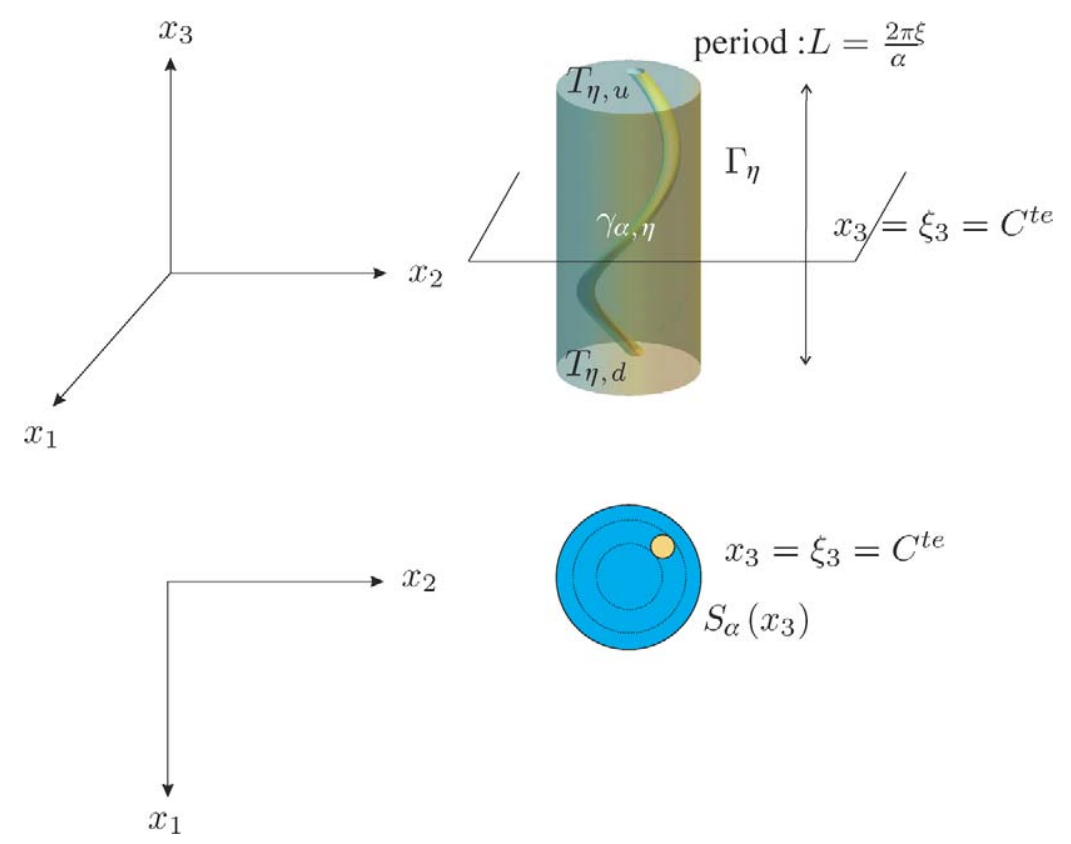

Fig. 1. A twisted helicoidal structure.

Fig. 1. Une structure torsadée hélicoïdale.

which describes the change in the material properties only depends on the first two variables $\xi_{1}$ and $\xi_{2}$. From a geometric point of view, the matrix $\mathbf{T}$ plays the role of the metric tensor. Another way to see it is that the change of coordinates amounts to replacing the different materials (often homogeneous and isotropic) by equivalent inhomogeneous anisotropic materials. It allows for a simple setting of the finite element method for twisted structures [2].

A twisted structure can now be defined as a structure whose characteristics depend only on the $\xi_{1}$ and $\xi_{2}$ coordinates, as described above.

Note that such a structure is invariant along $\xi_{3}$ but $\frac{2 \pi}{\alpha}$-periodic along $x_{3}$.

\section{Asymptotic algorithms for a weakly twisted structure}

\subsection{What is a weakly twisted structure?}

The problem is not as simple as it seems. In order to understand the difficulty, let us take an example coming from mechanics. Let us consider a bar of infinite extent turning with constant angular velocity $\omega$. Whatever small $\omega$ is, there exist some points of the bar, of sufficiently large cross-section, far away from the axis of rotation, which move with a velocity as large as one wants (restricted only by the size of the cross-section). As a result, the very motion of a weakly twisted fibre is linked with the fact that there exists a finite distance beyond which the problem is impervious to any rotation (being that the permittivity is constant beyond this distance or that there are boundary conditions at a finite distance). Let $D$ be the distance from the rotation axis to the most distant point not impervious to the rotation. Let $L=$ $\frac{2 \pi}{\alpha}$ be the length of a complete twist, i.e., the period of the helix. Also, let $\eta$ be a small parameter representing a ratio between the diameter of the cross-section of the helix to its period along the $x_{3}$ axis. Two points of view are possible:

- $D$ is kept fixed whereas $L_{\eta}$ depends on a parameter $\eta$ in the following way: $D$ fixed and $L_{\eta}=L / \eta$.

- $L$ is fixed and $D_{\eta}$ depends now on the parameter $\eta$ in the following way: $L$ is fixed and $D_{\eta}=\eta D$.

In this Note, the second point of view is assumed. This is the reason why we introduce the scaled variables in the cross-section. In the following, the asymptotic model for weakly twisted structures is found by letting $\eta \rightarrow 0$. The idea of the asymptotic approach for thin domains is similar to that of [3, Chapter 3]; however the helicoidal twist brings a new feature into the problem and its solution. 


\subsection{The asymptotic model}

Let us consider a sequence of bounded sets $\Omega_{\alpha, \eta}$ in $\mathbb{R}^{3}$ defined by $\Omega_{\alpha, \eta}=[0,2 \pi / \alpha] \times B\left(0, D_{\eta}\right)$, where $B\left(0, D_{\eta}\right)$ is the disk with the centre at $\left(0,0, x_{3}\right)$ and radius $D_{\eta} . \Omega_{\alpha, \eta}$ has a boundary $\partial \Omega_{\alpha, \eta}$ which consists of a (possibly thin) vertical wall $W_{\alpha, \eta}$ and two opposite (up and down) transverse sides $T_{u, \eta}$ and $T_{d, \eta}$ respectively in the planes $\left(x_{1}, x_{2}, 0\right)$ and $\left(x_{1}, x_{2}, 2 \pi / \alpha\right)$. We further assume that $W_{\alpha, \eta}$ consists of two parts, one of which $\Gamma_{\eta}$ is invariant along the $x_{3}$-axis, and the other one $\gamma_{\alpha, \eta}$ which depends upon the torsion $\alpha$. Altogether, this boundary $\partial \Omega_{\alpha, \eta}$ writes as $\partial \Omega_{\alpha, \eta}=W_{\alpha, \eta} \cup T_{\eta}=\Gamma_{\eta} \cup \gamma_{\alpha, \eta} \cup T_{\eta, u} \cup T_{\eta, d}$ (see Fig. 1). Let us now consider the following electrostatic problem:

$$
\left\{\begin{array}{l}
\nabla^{2} u_{\eta}=0 \text { in } \Omega_{\alpha, \eta} \\
\left.u_{\eta}\right|_{\Gamma_{\eta}}=1 \\
\left.u_{\eta}\right|_{\gamma_{\alpha, \eta}}=0 \\
\left.u_{\eta}\right|_{T_{\eta, u}}=\left.u_{\eta}\right|_{T_{\eta, d}},\left.\quad \frac{\partial u_{\eta}}{\partial x_{3}}\right|_{T_{\eta, u}}=\left.\frac{\partial u_{\eta}}{\partial x_{3}}\right|_{T_{\eta, d}}
\end{array}\right.
$$

We note that the last set of boundary conditions defines indeed the period of the structure: since this one is infinite along the $x_{3}$-axis, we shall simply assume that the field takes the same values when the helix makes the $2 \pi$ rotation.

We introduce the scaled variables in the cross-section $\left(y_{1}, y_{2}, x_{3}\right)=\left(\frac{x_{1}}{\eta}, \frac{x_{2}}{\eta}, x_{3}\right)$ so that

$$
\nabla_{x}^{2}=\frac{1}{\eta^{2}}\left(\frac{\partial^{2}}{\partial y_{1}^{2}}+\frac{\partial^{2}}{\partial y_{2}^{2}}\right)+\frac{\partial^{2}}{\partial x_{3}^{2}}:=\frac{1}{\eta^{2}} \nabla_{y}^{2}+\frac{\partial^{2}}{\partial x_{3}^{2}}
$$

The field $u_{\eta}$ is approximated by the following Ansatz

$$
u_{\eta} \sim u_{0}\left(\frac{x_{1}}{\eta}, \frac{x_{2}}{\eta}, x_{3}\right)+\eta^{2} u_{1}\left(\frac{x_{1}}{\eta}, \frac{x_{2}}{\eta}, x_{3}\right)
$$

Of course, the trajectory $\gamma_{\alpha, \eta}$ characterising the position of the inner boundary will depend on the $x_{3}$ coordinate via a small perturbation characterised by a smooth vector function $\mathbf{g}\left(\alpha, x_{3}\right)$ as follows

$$
\boldsymbol{\gamma}_{\alpha, \eta}\left(x_{1}, x_{2}, x_{3}\right)=\boldsymbol{\gamma}\left(x_{1}, x_{2}, 0\right)+\eta \mathbf{g}\left(\alpha, x_{3}\right)
$$

The value of the field on the boundary $\gamma_{\alpha, \eta}$ is therefore given by

$$
\left.u_{\eta}\right|_{\gamma_{\alpha, \eta}\left(x_{1}, x_{2}, x_{3}\right)}=\left.u_{\eta}\right|_{\gamma\left(x_{1}, x_{2}, 0\right)}+\left.\eta \mathbf{g}\left(\alpha, x_{3}\right) \cdot \nabla u_{\eta}\right|_{\gamma\left(x_{1}, x_{2}, 0\right)}
$$

Hence, from (7) and (9) we have

$$
\left.u_{\eta}\right|_{\gamma_{\alpha, \eta}\left(x_{1}, x_{2}, x_{3}\right)}=\left.u_{0}\right|_{\gamma\left(x_{1}, x_{2}, 0\right)}+\left.\eta^{2} u_{1}\right|_{\gamma\left(x_{1}, x_{2}, 0\right)}+\left.\eta \mathbf{g}\left(\alpha, x_{3}\right) \cdot \nabla_{x} u_{0}\right|_{\gamma\left(x_{1}, x_{2}, 0\right)}+\left.\eta^{3} \mathbf{g}\left(\alpha, x_{3}\right) \cdot \nabla_{x} u_{1}\right|_{\gamma\left(x_{1}, x_{2}, 0\right)}
$$

It follows from (5), (7) and (10) that $u_{0}$ is a solution of the Dirichlet problem

$$
\left\{\begin{array}{l}
\nabla_{y}^{2} u_{0}\left(y_{1}, y_{2}, x_{3}\right)=0 \quad \text { in } S_{\alpha}\left(x_{3}\right) \\
\left.u_{0}\right|_{\Gamma}=1 \\
\left.u_{0}\right|_{\gamma\left(x_{3}\right)}=0
\end{array}\right.
$$

where $S_{\alpha}\left(x_{3}\right)$ is the two-dimensional trace of $\Omega_{\alpha, \eta}$ on a constant $x_{3}$-plane and which corresponds to the leading order approximation. We can now iterate and collect terms of higher order to deduce

$$
\left\{\begin{array}{l}
\nabla_{y}^{2} u_{1}\left(y_{1}, y_{2}, x_{3}\right)+\frac{\partial^{2} u_{0}}{\partial x_{3}^{2}}=0 \quad \text { in } S_{\alpha}\left(x_{3}\right) \\
\left.u_{1}\right|_{\Gamma}=0 \\
\left.u_{1}\right|_{\gamma\left(x_{3}\right)}=0
\end{array}\right.
$$

From (1), the derivation with respect to $x_{3}$ in a twisted structure can be expressed via an angular momentum operator in the cross-section $\frac{\partial .}{\partial x_{3}}=\alpha\left(x_{1} \frac{\partial}{\partial x_{2}}-x_{2} \frac{\partial .}{\partial x_{1}}\right):=\alpha \mathcal{L}($.$) . Note that this operator is independent of the scaling: \mathcal{L}()=$. $y_{1} \frac{\partial .}{\partial y_{2}}-y_{2} \frac{\partial \cdot}{\partial y_{1}}=\frac{x_{1}}{\eta} \frac{\partial .}{\partial x_{2} / \eta}-\frac{x_{2}}{\eta} \frac{\partial .}{\partial x_{1} / \eta}=x_{1} \frac{\partial \cdot}{\partial x_{2}}-x_{2} \frac{\partial .}{\partial x_{1}}$. 


\subsection{Numerical setting of the asymptotic model}

A convenient way to deal with the numerical computation of the corrector is to work in unscaled two-dimensional co-ordinates. Let us introduce the auxiliary functions independent of $\eta$ :

$U_{0}\left(x_{1}, x_{2}\right)=u_{0}\left(x_{1} / \eta, x_{2} / \eta, 0\right)=u_{0}\left(y_{1}, y_{2}, 0\right)$ and $U_{1}\left(x_{1}, x_{2}\right)=\eta^{2} u_{1}\left(x_{1} / \eta, x_{2} / \eta, 0\right)=\eta^{2} u_{1}\left(y_{1}, y_{2}, 0\right)$.

It follows immediately that $U_{0}$ and $U_{1}$ are solutions of the problems:

$$
\left\{\begin{array}{l}
\nabla_{x}^{2} U_{0}\left(x_{1}, x_{2}\right)=0 \quad \text { in } S_{\alpha}(0) \\
\left.U_{0}\right|_{\Gamma}=1 \\
\left.U_{0}\right|_{\gamma(0)}=0
\end{array}\right.
$$

and

$$
\left\{\begin{array}{l}
\nabla_{x}^{2} U_{1}\left(x_{1}, x_{2}\right)+\alpha^{2} \mathcal{L}^{2}\left(U_{0}\right)=0 \quad \text { in } S_{\alpha}(0) \\
\left.U_{1}\right|_{\Gamma}=0 \\
\left.U_{1}\right|_{\gamma(0)}=0
\end{array}\right.
$$

These are well defined two-dimensional problems independent of the scaling. Note that the $\alpha^{2} \mathcal{L}^{2}\left(U_{0}\right)$ is an equivalent 'volume' source. As the boundary conditions in (14) are homogeneous, $U_{1}$ is proportional to this source term. Therefore, it is sufficient to compute the corrector for $\alpha=1$ and to multiply the result by the actual value of $\alpha^{2}$.

The next step is to give the weak form of the term $\mathcal{L}^{2}\left(U_{0}\right)$ in order to get a finite element formulation for the corrector. The $\mathcal{L}($.) operator can be expressed as

$$
\mathcal{L}(u)=\mathbf{R} \cdot \operatorname{grad} u
$$

with $\mathbf{R}=\underline{\underline{R}}_{\pi / 2} \mathbf{r}$ where $\underline{\underline{R}}_{\pi / 2}=\left(\begin{array}{cc}0 & -1 \\ 1 & 0\end{array}\right)$ is the $\pi / 2$ counterclockwise rotation matrix and $\mathbf{r}$ the position vector in the plane $\left(\begin{array}{l}x_{1} \\ x_{2}\end{array}\right)$ in rectangular coordinates. One has

$$
\operatorname{div}(\mathbf{R} u)=\mathbf{R} \cdot \operatorname{grad} u+u \operatorname{div} \mathbf{R}=\mathcal{L}(u)
$$

for any function $u$ since $\operatorname{div} \mathbf{R}=0$ and

$$
\operatorname{div}(\mathbf{R} u v)=\operatorname{div}(\mathbf{R} u) v+\mathbf{R} u \cdot \operatorname{grad} v=\mathcal{L}(u) v+u \mathcal{L}(v)
$$

for any functions $u$ and $v$.

The source term in $(14)$ is $\mathcal{L}^{2}\left(U_{0}\right)$. Let $u$ denotes $\mathcal{L}\left(U_{0}\right)$ in the sequel so that the contribution of the $\mathcal{L}^{2}\left(U_{0}\right)=\mathcal{L}(u)$ term in the weak formulation is given by

$$
\int_{S} \mathcal{L}(u) v \mathrm{~d} x_{1} \mathrm{~d} x_{2}=\int_{S} \operatorname{div}(\mathbf{R} u v) \mathrm{d} x_{1} \mathrm{~d} x_{2}-\int_{S} \mathbf{R} u \cdot \operatorname{grad} v \mathrm{~d} x_{1} \mathrm{~d} x_{2}
$$

where $v$ is the weighting function. By the divergence theorem, the first term is

$$
\int_{S} \operatorname{div}(\mathbf{R} u v) \mathrm{d} x_{1} \mathrm{~d} x_{2}=\int_{\partial S} \mathbf{R} u v \cdot \mathbf{n} \mathrm{d} l=0
$$

since $v$ can be taken equal to zero on the Dirichlet condition boundaries. Therefore $\int_{S} \mathcal{L}(u) v \mathrm{~d} x_{1} \mathrm{~d} x_{2}=-\int_{S} u \mathbf{R}$. $\operatorname{grad} v \mathrm{~d} x_{1} \mathrm{~d} x_{2}=-\int_{S} u \mathcal{L}(v) \mathrm{d} x_{1} \mathrm{~d} x_{2}$. It means that $\mathrm{i} \mathcal{L}$ is symmetric and that is not a surprise since $\mathrm{i} \mathcal{L}$ is an angular momentum observable in quantum mechanics and is therefore self-adjoint when acting in $L^{2}\left(\mathbb{R}^{2}\right)$. The weak form for the contribution is therefore:

$$
\int_{S} \mathcal{L}^{2}\left(U_{0}\right) v \mathrm{~d} x_{1} \mathrm{~d} x_{2}=-\int_{S} \mathcal{L}\left(U_{0}\right) \mathcal{L}(v) \mathrm{d} x_{1} \mathrm{~d} x_{2}
$$

and the weak form of (14) which gives the finite element formulation for $U_{1}$ is

$$
\int_{S}\left(\operatorname{grad} U_{0} \cdot \operatorname{grad} v+\alpha^{2} \mathcal{L}\left(U_{0}\right) \mathcal{L}(v)\right) \mathrm{d} x_{1} \mathrm{~d} x_{2}=0
$$

The asymptotic approximation of the solution of the twisted problem in the $x_{3}=0$ plane is simply given by $U_{0}+U_{1}$. 


\subsection{A numerical example}

We consider the structure the cross-section of which is presented on Fig. 2: an outer cylinder (radius $R=0.2 \mathrm{~m}$ ), the axis of which is the rotation axis of the twisted structure, and an inner spiral with a circular cross-section (radius $a=0.05 \mathrm{~m}$ and its center is $0.1 \mathrm{~m}$ from the rotation axis). In this case $D=0.2, L=2 \pi / \alpha$ and the 'small parameter' is $D / L=1.26 \alpha$. Figs. 2 and 3 show the electrostatic field and the asymptotic corrector for the case of $\alpha=2 \pi$. Table 1 presents the results of comparison of asymptotic approximations and finite element numerical data; it gives the variation of the error at a given point within the domain for the asymptotic prediction as a function of the twist $\alpha$. The first column is the value of the twist parameter $\alpha$ therefore the first line $\alpha=0$ corresponds to the untwisted case and the following lines correspond to increasingly twisted cases. The second column corresponds to an exact
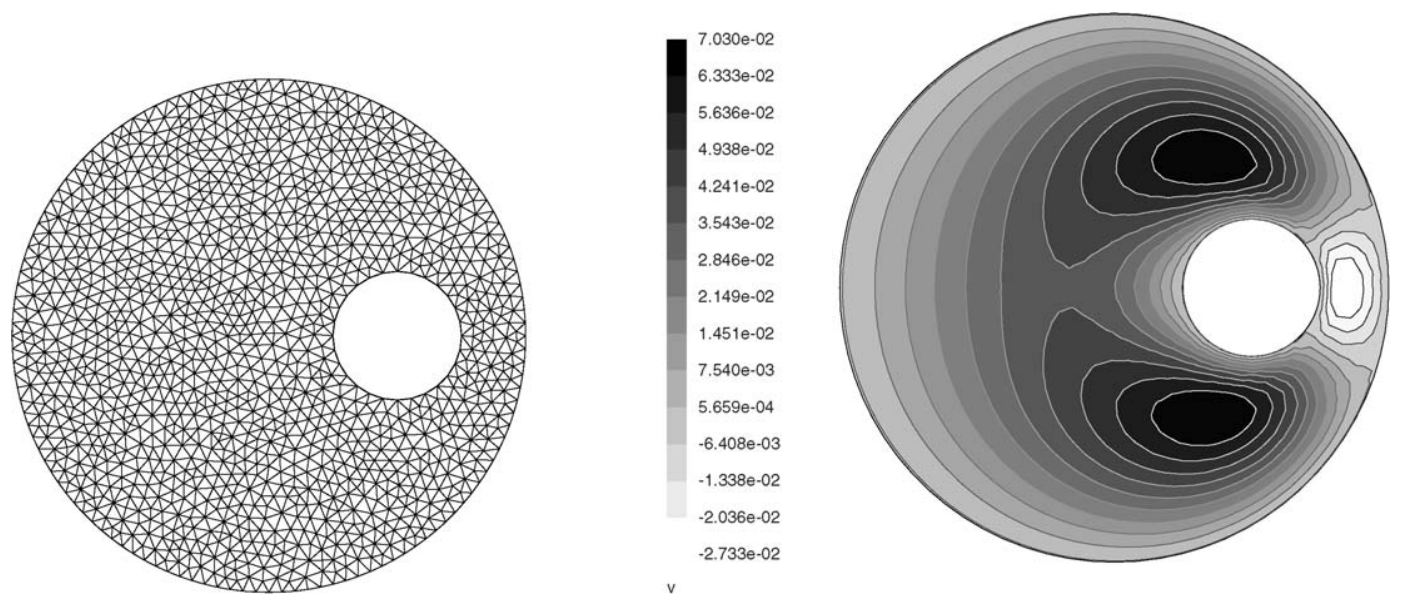

Fig. 2. Cross-section of the structure with its finite element mesh (left) and an isovalue map of the corrector $U_{1}$ for $\alpha=2 \pi$ (right).

Fig. 2. Section droite de la structure avec son maillage en éléments finis (gauche) et une carte des isovaleurs du correcteur $U_{1}$ pour $\alpha=2 \pi$ (droite).
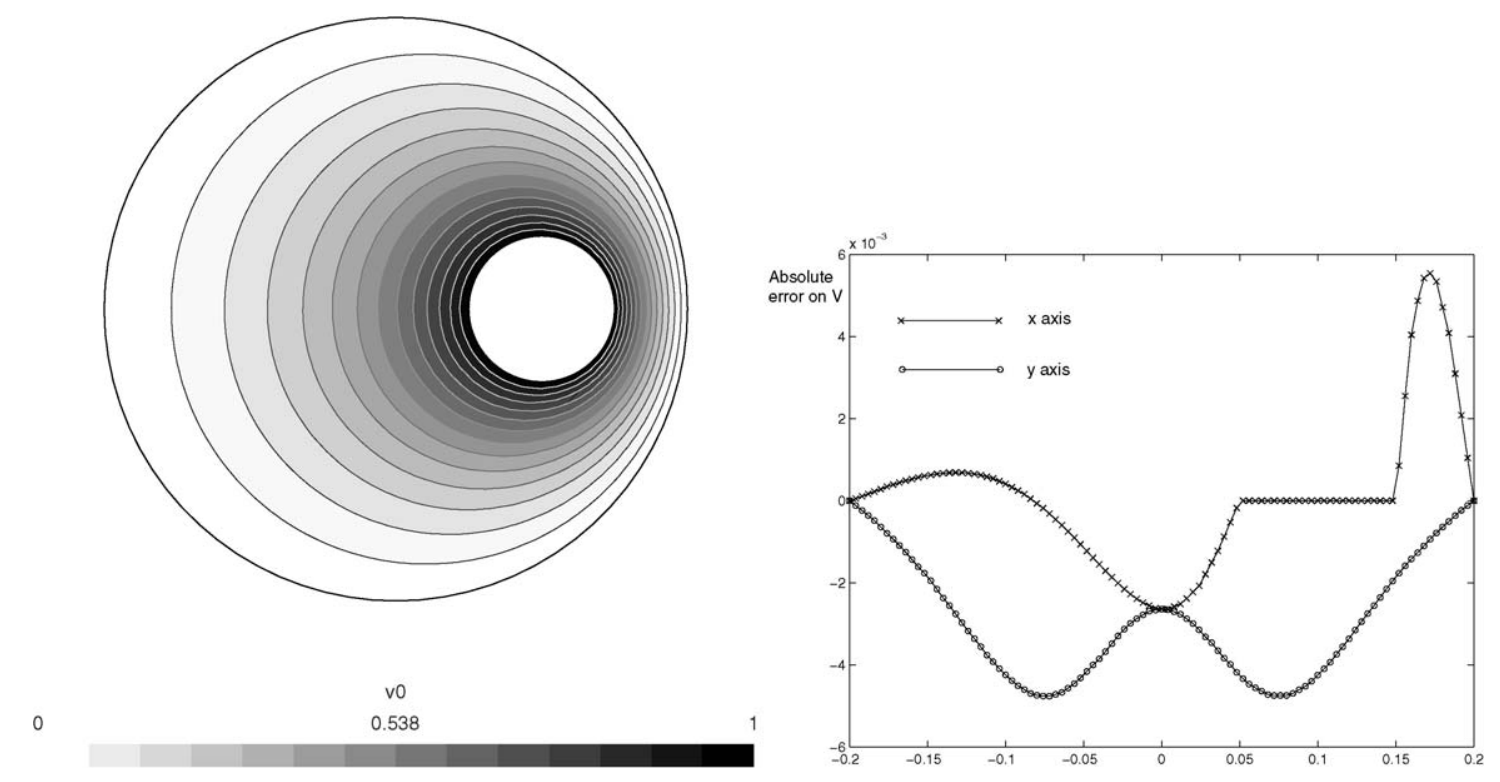

Fig. 3. Isovalue map of $U_{0}$ corresponding to the untwisted problem (left) and plots of the error variation along the axes between the twisted problem and the asymptotic estimation $U_{0}+U_{1}$ for $\alpha=2 \pi$ (right).

Fig. 3. Carte des isovaleurs de $U_{0}$ correspondant au problème non torsadé (gauche) et courbes de la variation de l'erreur suivant les axes, entre le problème torsadé et l'approximation asymptotique $U_{0}+U_{1}$ pour $\alpha=2 \pi$ (droite). 
Table 1

Comparison of the values of the potential at point $(0.175,0)$ (the origin is on the rotation axis) where the error is the most important. On this point $U_{1}=-6.54036087 \mathrm{e}-004$ and $U_{0}=0.4323075525521577$

Tableau 1

Comparaison des valeurs du potentiel au point $(0.175,0)$ (l'origine est sur l'axe de rotation) où l'erreur est la plus importante. En ce point, $U_{1}=-6,54036087 \mathrm{e}-004$ et $U_{0}=0,4323075525521577$

\begin{tabular}{lllll}
\hline$\alpha$ & $u$ exact & $u$ asymptotic & Abs. error & $\alpha^{2} U_{1}$ correction \\
\hline 0 & 0.4323075525521577 & & & \\
0.1 & 0.4323010126383421 & 0.43230101219128 & $4.4706 \mathrm{e}-010$ & $-6.5404 \mathrm{e}-006$ \\
0.5 & 0.4321443225126496 & 0.43214404353032 & $2.7898 \mathrm{e}-007$ & $-1.6351 \mathrm{e}-004$ \\
1 & 0.431657958832633 & 0.43165351646482 & $4.4424 \mathrm{e}-006$ & $-6.5404 \mathrm{e}-004$ \\
2 & 0.4297611380445051 & 0.42969140820282 & $6.9730 \mathrm{e}-005$ & $-2.6161 \mathrm{e}-003$ \\
3 & 0.4267632298417439 & 0.42642122776615 & $3.4200 \mathrm{e}-004$ & $-5.8863 \mathrm{e}-003$ \\
5 & 0.4183500933323935 & 0.41595665036880 & $2.3934 \mathrm{e}-003$ & $-1.6351 \mathrm{e}-002$ \\
8 & 0.403178325558716 & 0.39044924296276 & $1.2729 \mathrm{e}-002$ & $-4.1858 \mathrm{e}-002$ \\
10 & 0.3933602365265181 & 0.36690394381872 & $2.6456 \mathrm{e}-002$ & $-6.5404 \mathrm{e}-002$ \\
20 & 0.3617484871340219 & 0.17069311761841 & $1.9106 \mathrm{e}-001$ & $-2.6161 \mathrm{e}-001$ \\
\hline
\end{tabular}

model, i.e., a two-dimensional finite element model with equivalent material properties [1,2] involving the $T$ matrix given by Eq. (4). Finite element numerical computations have been performed with GetDD [4]. The third column gives our asymptotic approximation. The fourth column gives the discrepancy between the two previous columns. The fifth column gives the value of the asymptotic correction to be added to the untwisted case $\alpha=0$ to obtain the third column. Even if this value is small, a relatively large value with respect to the fourth column indicates that the asymptotic correction is nevertheless substantial.

This shows that the asymptotic formula provides a good accuracy for small twists, i.e., small values of $\alpha$, and it also gives correct qualitative predictions for a wider range of admissible values of $\alpha$.

\section{Conclusion}

In this Note, we have presented an analysis of weakly twisted structures in the case of an electrostatic model problem. Via the asymptotic procedure, we have reduced a full three-dimensional formulation to a sequence of twodimensional problems on a scaled, parameter independent domain. This makes the numerical study efficient and fast. The asymptotic algorithm described here was checked against rigorous finite element computations. An extension of this study is the analysis of dispersion relations of weakly twisted waveguides in the case of a time harmonic electromagnetic eigenvalue problem.

\section{Acknowledgements}

S. Guenneau acknowledges support from DoD/ONR MURI grant N00014-01-1-0803 during his time at Imperial College London in 2003-2004 and a visiting professorship at Université Aix-Marseille III in July 2005. This work was also supported by the Franco-British Partnership Programme ALLIANCE (Project PN 05.026). The authors wish to thank Dr. N.V. Movchan and Prof. R.C. McPhedran for related discussions.

\section{References}

[1] F. Zolla, G. Renversez, A. Nicolet, B. Kuhlmey, S. Guenneau, D. Felbacq, Foundations of Photonic Crystal Fibres, Imperial College Press, London, 2005.

[2] A. Nicolet, F. Zolla, S. Guenneau, Modelling of twisted optical waveguides with edge elements, Eur. Phys. J. Appl. Phys. 28 (2004) 153-157, doi:10.1051/epjap:2004189.

[3] A.B. Movchan, N.V. Movchan, Mathematical Modelling of Solids with Non-Regular Boundaries, CRC Press, Boca Raton, FL, 1995.

[4] P. Dular, C. Geuzaine, F. Henrotte, W. Legros, A general environment for the treatment of discrete problems and its application to the finite element method, IEEE Trans. Magnetics 34 (5) (1998) 3395-3398, and see also the Internet address http://www.geuz.org. 